\title{
Cinémas
}

Revue d'études cinématographiques

Journal of Film Studies

\section{Femme active et femme au foyer dans le woman's horror film américain des années 2000 : ambivalences, contradictions et impasse post-féministe}

\section{The Active Woman and the Stay-at-Home Woman in the "Woman's Horror Film" of the 2000s in the United States: Ambivalences, Contradictions and Post-Feminist Impasse}

Pascale Fakhry

Volume 22, numéro 2-3, printemps 2012

Genre/Gender

URI : https://id.erudit.org/iderudit/1011655ar

DOI : https://doi.org/10.7202/1011655ar

Aller au sommaire du numéro

Éditeur(s)

Cinémas

ISSN

1181-6945 (imprimé)

1705-6500 (numérique)

Découvrir la revue

Citer cet article

Fakhry, P. (2012). Femme active et femme au foyer dans le woman's horror film américain des années 2000 : ambivalences, contradictions et impasse post-féministe. Cinémas, 22(2-3), 61-79. https://doi.org/10.7202/1011655ar
Résumé de l'article

Les années 2000 voient la sortie d'un grand nombre de films d'horreur qui sont aussi des woman's films. Ces oeuvres montrent une femme confrontée à des créatures monstrueuses, souvent d'origine surnaturelle, et à des problèmes spécifiquement féminins, tels que la maternité et le conflit travail/foyer. À travers l'analyse détaillée de deux woman's horror films de l'époque - The Ring (Gore Verbinski, 2002) et The Others (Alejandro Amenábar, 2001) -, l'auteure se propose de comparer, dans un premier temps, le traitement réservé à la femme active et à la femme au foyer dans leur relation à la maternité et aux rôles de victime, de monstre ou d'héroïne qu'on leur attribue au sein du récit d'horreur. L'ambivalence du discours tenu par le woman's horror film sur ses personnages principaux féminins est, dans un deuxième temps, mise en relation avec l'émergence du post-féminisme et son influence grandissante sur la culture populaire américaine des années 2000 . 


\title{
Femme active et femme au foyer dans le woman's horror film américain des années 2000 : ambivalences, contradictions et impasse post-féministe
}

\section{Pascale Fakhry}

\begin{abstract}
RÉSUMÉ
Les années 2000 voient la sortie d'un grand nombre de films d'horreur qui sont aussi des woman's films. Ces œuvres montrent une femme confrontée à des créatures monstrueuses, souvent d'origine surnaturelle, et à des problèmes spécifiquement féminins, tels que la maternité et le conflit travail/foyer. À travers l'analyse détaillée de deux woman's horror films de l'époque - The Ring (Gore Verbinski, 2002) et The Others (Alejandro Amenábar, 2001) —, l'auteure se propose de comparer, dans un premier temps, le traitement réservé à la femme active et à la femme au foyer dans leur relation à la maternité et aux rôles de victime, de monstre ou d'héroïne qu'on leur attribue au sein du récit d'horreur. L'ambivalence du discours tenu par le woman's horror film sur ses personnages principaux féminins est, dans un deuxième temps, mise en relation avec l'émergence du postféminisme et son influence grandissante sur la culture populaire américaine des années 2000.
\end{abstract}

Les années 2000 voient la sortie d'un grand nombre de films d'horreur hollywoodiens centrés sur un personnage principal féminin. C'est le cas de Bless the Child (Chuck Russel, 2000), The Cell (Tarsem Singh, 2000), What Lies Beneath (Robert Zemeckis, 2000), The Gift (Sam Raimi, 2000), The Others (Alejandro Amenábar, 2001), Abandon (Stephen Gaghan, 2002), The Ring (Gore Verbinski, 2002), They (Robert Harmon, 2002), Gothika (Mathieu Kassovitz, 2003), The Grudge (Takashi Shimizu, 2004), Toolbox Murders (Tobe 
Hooper, 2004), Dark Water (Walter Salles, 2005), The Ring Two (Hideo Nakata, 2005), The Skeleton Key (Iain Softley, 2005), The Reaping (Stephen Hopkins, 2007), Paranormal Activity (Oren Peli, 2007), 100 Feet (Eric Red, 2008), Drag Me to Hell (Sam Raimi, 2009), etc. Les protagonistes féminines de ces films sont généralement interprétées par des stars ou des comédiennes de renom: Jennifer Lopez dans The Cell, Kim Basinger dans Bless the Child, Cate Blanchett dans The Gift, Michelle Pfeiffer dans What Lies Beneath, Nicole Kidman dans The Others, Halle Berry et Penélope Cruz dans Gothika, Naomi Watts dans The Ring, Sarah Michelle Gellar dans The Grudge, etc. Dans tous les cas, ces protagonistes sont confrontées à la fois à des créatures monstrueuses, souvent d'origine surnaturelle et qui mettent leur vie en danger, et à des problèmes liés à leur identité féminine: une maternité problématique (Bless the Child, The Cell, The Ring et The Ring Two, The Others, Dark Water, The Reaping, etc.) ou un conflit travail/foyer (Abandon, The Toolbox Murder, The Grudge, The Skeleton Key, Drag Me to Hell, etc.). Bien que ces films soient des films d'horreur, ce sont également des woman's films, parce qu'ils placent "au centre de [leur] univers une femme qui essaye de faire face à des problèmes émotionnels, sociaux et psychologiques qui sont spécifiquement liés au fait d'être femme ${ }^{1} »$ (Basinger 1995, p. 20).

La tradition du woman's horror film remonte à la fin des années 1960 : comme l'a montré Andrew Tudor (1989, p. 108121), dans la plupart des films d'horreur sortis entre 1931 et 1960, les personnages féminins tenaient un rôle périphérique et leurs problèmes et désirs féminins ne fonctionnaient pas comme moteur de l'action. Les rôles du monstre - dont les désirs destructeurs déclenchent l'intrigue - et du héros, ou de l'«expert», comme l'appelle Tudor - dont l'intervention vise à clore le récit - sont en majeure partie interprétés par des comédiens masculins. Dans le film d'horreur de l'époque, en revanche, «il est rare de trouver des femmes occupant un rôle narratif et social en dehors de celui de victime ${ }^{2} "$ (p. 126). Les personnages actifs et masculins sont, en outre, ceux auxquels le récit accorde la plus grande profondeur psychologique et sur qui se concentre l'implication spectatorielle. Les victimes, «citoyens 
de seconde classe du genre", toujours selon Tudor, n'existent que pour «fournir le terrain humain sur lequel monstres et experts, menace et défense, pulsions de désordre et d'ordre peuvent s'affronter ${ }^{3} »($ p. 119).

Rosemary's Baby de Roman Polanski (1968) est l'un des premiers films qui vient bouleverser ce schéma en plaçant au centre de son univers une jeune femme et en racontant, de son point de vue, son histoire: Rosemary (Mia Farrow) est violée et fécondée, à son insu, par le diable. Le film explore la question de la relation de son personnage principal féminin à sa maternité et à son corps, dont les transformations au cours de la grossesse sont dépeintes comme inquiétantes et monstrueuses. Il examine, par ailleurs, la nature du rapport de Rosemary à sa fonction de femme au foyer. La jeune femme est représentée comme la victime de la division traditionnelle des rôles entre les sexes: Guy (John Cassavetes), son mari, est l'unique pourvoyeur des besoins matériels du couple et, pour cela, considère sa femme comme un objet dont il est le propriétaire. Il vend son corps au diable en échange d'un rôle dans une pièce de théâtre. En faisant de Guy le complice de Satan, le film diabolise l'autorité que l'institution du mariage et le monopole du pouvoir financier attribuent aux hommes. Sorti en 1968, Rosemary's Baby s'inspire manifestement du discours naissant de la deuxième vague de féminisme qui, depuis la publication en 1963 du bestseller de Betty Friedan, The Feminine Mystique ${ }^{4}$, regarde la division traditionnelle des rôles entre les sexes et la fonction de femme au foyer comme des instruments d'oppression des femmes.

Rosemary's Baby donne naissance à un cycle de woman's horror films qui sortent dans les années 1970 et qui se centrent sur un personnage féminin confronté à la fois à un monstre masculin et à des problèmes féminins liés à sa maternité ou à ses rôles sociaux. C'est le cas de Sisters (Brian De Palma, 1973), The Stepford Wives (Bryan Forbes, 1975), Demon Seed (Donald Cammell, 1977), Eyes of Laura Mars (Irvin Kershner, 1978), The Entity (Sidney J. Furie, 1982), etc. Comme Rosemary's Baby, ces films, Sisters et The Stepford Wives en particulier, sont considérés comme profondément imprégnés du discours de la deuxième vague de féminisme ${ }^{5}$. La vague de réactions contre le

Femme active et femme au foyer dans le woman's horror film américain des années 2000 : ambivalences, contradictions et impasse post-féministe 
féminisme dans les années 1980 et 1990 (Faludi 1991) entraîne la disparition du woman's horror film du paysage générique du film d'horreur.

Le sous-genre refait cependant surface dans les années 2000, à une époque considérée comme "post-féministe", et où on estime que les revendications égalitaires des féministes de la deuxième vague ont été entendues et que leur discours est "dépassé». Quel sens attribuer à la renaissance du woman's horror film dans ce contexte socioculturel et comment expliquer, à la lumière du post-féminisme, l'insistance du sous-genre à construire ses personnages principaux féminins en fonction de leur relation à la maternité et au conflit travail/foyer?

Nous tenterons de répondre à ces questions à travers l'analyse de deux woman's horror films: The Ring et The Others. Notre choix s'est porté sur ces deux films à cause de leur succès ${ }^{6}$ et parce que, comme nous le montrerons dans cet article, le traitement qu'ils réservent à leurs personnages féminins dans leur rapport aux rôles de victime, de monstre ou d'héroïne et aux fonctions de femme active et de femme au foyer est représentatif du discours tenu par le sous-genre, dans les années 2000, sur ces questions.

\section{The Ring: femme active, maternité sacrificielle et héroïsme}

Rachel (Naomi Watts), le personnage principal féminin de The Ring, est une mère célibataire et une journaliste ambitieuse qui enquête sur la mort mystérieuse de sa nièce et de ses amis dans l'espoir d'écrire un article à sensation. Ces adolescents sont, en effet, tous morts le même jour à la même heure, une semaine exactement après avoir visionné une cassette vidéo au contenu étrange. La jeune femme trouve la cassette en question, la regarde, et reçoit immédiatement après un coup de fil la prévenant qu'il ne lui reste plus qu'une semaine à vivre. Non seulement Rachel devient ainsi une victime potentielle à cause de son métier et de son ambition professionnelle, mais l'importance qu'elle accorde à son travail fait d'elle une mère négligente: dès sa première apparition à l'écran, la jeune femme, parce qu'elle est en pleine conversation téléphonique avec son chef, arrive en retard pour prendre son fils, Aidan (David Dorfman), à la sortie 
de l'école. Plus tard, Rachel, préoccupée par son enquête, ne se lève pas avec le garçon le matin et le laisse préparer seul ses sandwichs avant d'aller à l'école. Parce qu'elle est une femme active, Rachel est représentée comme une mauvaise mère, une mère dont le travail met la vie de son fils en danger: Aidan découvre un soir la cassette vidéo maudite que Rachel a rapportée à la maison et la visionne. Cet incident transforme la jeune femme. Son but devient, dès lors, de résoudre l'énigme de la vidéo, non plus pour satisfaire son ambition professionnelle, mais pour sauver la vie de son fils. Son enquête la mène sur les traces de Samara, une petite fille morte qui serait à l'origine du film maléfique, et un accident la précipite jusqu'au fond du puits où se trouvent les restes de l'enfant-monstre. Du fond de sa tombe, Samara lui révèle son histoire. Rachel apprend que la fillette a été assassinée par sa propre mère et qu'elle aurait créé le film qui tue en enregistrant ses pensées après sa mort sur une pellicule vidéo afin de se venger du sort qui lui a été fait. En tombant dans le puits où le cadavre de Samara repose, Rachel se révèle disposée à donner sa vie pour préserver celle de son fils et, pour cela, parvient à percer le mystère de la vidéo.

Le récit de The Ring se construit autour de la transformation de Rachel de mauvaise en bonne mère et de victime en héroïne. Rachel sauve Aidan en se révélant prête à renoncer non seulement à sa vie pour lui, mais aussi à sa carrière (au début de The Ring Two, le sequel de The Ring, nous apprenons même que la jeune femme a cessé de travailler pour le Seattle Post, s'est installée avec son fils dans une petite ville où elle est employée par un petit journal local, ce qui lui donne le temps de se consacrer davantage à Aidan). The Ring associe le statut de victime de Rachel et sa négligence envers Aidan à son état de femme active et ambitieuse. Il établit, de plus, un rapport d'équivalence entre l'hérö̈sme féminin, la maternité sacrificielle et la capacité de son personnage principal à renoncer à sa carrière pour le bien de son enfant. The Ring réserve le même type de traitement à la femme active dans son rapport à sa maternité que Ringu (Hideo Nakata, 1998), le film japonais dont il est le remake.

L'histoire de Rachel reprend de manière relativement fidèle celle de Reiko (Nanako Matsushima), le personnage principal 
féminin de Ringu. Reiko est, en effet, une journaliste ambitieuse qui a peu de temps à consacrer à son fils et dont l'enquête sur la vidéo maléfique met sa vie et celle de Yôichi (Rikiya Ôtaka) en danger. Pour sauver son fils, elle doit apprendre à devenir une bonne mère. Comme le montre Ruth Goldberg (2004, p. 380), la descente de Reiko au fond du puits où elle trouve les restes de Sadako (Orie Izuno), la fille monstrueuse, scelle sa transformation en mère sacrificielle et enclenche son processus de rédemption. Comme The Ring, Ringu prône un idéal de maternité sacrificielle et suggère aux mères qui travaillent que le salut de leurs enfants dépend de leur disposition à renoncer symboliquement et littéralement à leur vie pour eux.

Pour Nina K. Martin (2008), le discours dépréciatif tenu par le film d'horreur japonais en général, et par Ringu en particulier, sur les mères célibataires qui travaillent serait emblématique de la peur que l'arrivée massive des femmes sur le marché du travail dans les années 1990 suscite dans la société nippone. Ces films seraient l'expression d'une nostalgie pour un passé imaginaire ou légendaire désormais révolu où l'attachement exclusif des femmes à leurs rôles traditionnels de femme au foyer et de mère était garant de l'ordre social. Toutefois, alors que les propos tenus par Ringu sur la femme active peuvent parâtre compréhensibles dans le contexte culturel de production du film, ils sont a priori plus surprenants dans son remake américain. Les remakes hollywoodiens de films étrangers ont, en effet, généralement tendance à traduire ou à trouver un équivalent culturel aux éléments du film original qui peuvent parâtre "étrangers" à la culture américaine ou en contradiction avec elle (Moine 2007, p. 102-107). Pourtant, bien que la majorité des femmes aient une activité professionnelle et que leur intégration au marché du travail ne soit pas un phénomène nouveau dans les États-Unis des années $2000^{7}$, les woman's horror films américains sont fidèles aux films d'horreur japonais, dont ils sont souvent les remakes dans le traitement réservé à la femme active.

Comme The Ring et Ringu, Dark Water et Honogurai mizu no soko kara (Hideo Nakata, 2002), le film japonais dont Dark Water est le remake, se centrent tous les deux sur une mère célibataire qui travaille. Yoshimi (Hitomi Kuroki) dans le film 
original japonais, Dahlia (Jennifer Connelly) dans son remake américain, doit trouver un emploi pour obtenir la garde de sa fille et, malgré tous les efforts qu'elle fait pour être une "bonne» mère, elle arrive souvent en retard pour prendre son enfant de l'école à cause de ses horaires de travail. Le monstre, dans les deux films, est en outre une petite fille qui est morte à cause de la négligence de sa mère, qui l'a laissée se promener seule sur le toit de l'immeuble où elles habitaient, près du réservoir d'eau. Depuis, tout ce que le fantôme de la fillette veut est une mère qui se consacre entièrement à elle. Son choix se porte sur Yoshimi/Dahlia et, quand celle-ci refuse de quitter sa propre fille pour elle, elle menace de tuer sa rivale. Afin de sauver sa fille, Yoshimi/Dahlia accepte de mourir. De mère célibataire et active forcée de négliger sa fille pour son travail, Yoshimi/Dahlia devient à la fin du film une mère et une héroïne sacrificielle. L'insistance des remakes américains à reproduire fidèlement les propos dépréciatifs tenus par les films japonais originaux sur la femme et la mère qui travaillent indique que ce trait est considéré, aux États-Unis, comme culturellement significatif. En effet, dans un grand nombre de woman's horror films hollywoodiens des années 2000 qui ne sont pas des remakes de films japonais, tels que The Reaping, The Skeleton Key ou Drag Me to Hell, le métier et l'ambition professionnelle du personnage féminin sont directement responsables de sa rencontre avec le monstre et de sa transformation en victime. Pour Martin (2008), les ressemblances entre les discours américain et japonais sur la relation des personnages principaux féminins du film d'horreur à leur métier et à leur maternité seraient représentatives d'une réaction globale contre les femmes actives. Toutefois, les woman's horror films hollywoodiens des années 2000 se distinguent des films d'horreur japonais de la même époque par le traitement qu'ils réservent à la femme au foyer.

\section{Mère déficiente et mère monstrueuse}

The Ring et Ringu comparent tous les deux leur victimel héroïne à la mère de la petite fille monstrueuse, une femme dont le comportement criminel est responsable de la métamorphose de son enfant en monstre. Dans Ringu, l'enquête de 
Reiko la met sur les traces de Sadako et de sa mère: la fillemonstre serait née de la relation adultère entre une sorcière, Shizuko (Masako), et un professeur d'université; elle a été abandonnée par sa mère qui s'est suicidée, et tuée par son père, effrayé par ses pouvoirs monstrueux - Sadako peut tuer par la simple force de sa volonté. Le film semble nous dire que les mères qui transgressent par leur comportement l'ordre social et qui abandonnent leurs enfants sont des monstres qui produisent des monstres. Dans The Ring, par contre, Samara a grandi au sein d'une famille unie et traditionnelle. Anna Morgan (Shannon Cochran), sa mère, était une femme au foyer et vivait avec son mari dans un ranch sur une île. Selon les termes employés par Rachel, Anna aurait mené une vie "confortable", "coupée du monde» et "surprotégée ${ }^{8}$ " dont le caractère traditionnel est mis en valeur par son costume: Anna est toujours montrée vêtue d'une robe noire qui semble venue d'un autre siècle. Son unique désir était d'avoir un enfant et de réaliser ce que la société patriarcale considère comme son destin de femme. Échouant à être enceinte, elle finit par adopter une petite fille qui, s'avère-t-il plus tard, souffre de troubles psychologiques graves. En devenant mère, Anna sombre dans la dépression et, selon son médecin, la docteure Grasnik (Jane Alexander), elle se met à avoir des visions horribles dès qu' elle se trouve à proximité de sa fille. De plus, la docteure Grasnik considère Anna comme une mauvaise mère, qui aurait pu sauver sa fille des troubles dont elle souffrait si elle l'avait aimée. Anna est dépeinte comme la victime d'une conception patriarcale de la féminité qui lui dit que son désir le plus cher est d'avoir un enfant qu'elle doit aimer inconditionnellement. Quand elle comprend qu'elle est incapable de se plier à cet idéal de maternité, Anna se transforme en monstre, tue sa fille et se suicide. La mère monstrueuse dans The Ring n'est pas la femme transgressive, mais la femme au foyer.

Bien que The Ring tienne un discours dépréciatif sur la femme active, il ne valorise pas pour autant la féminité et la famille traditionnelles. Au contraire, il considère la femme au foyer comme une mère plus mauvaise que la femme active, qui est simplement une mère déficiente. Il la regarde comme une victime qui risque de perdre la tête et de devenir un monstre si 
elle ne parvient pas à se plier à un idéal de maternité qui lui impose un amour inconditionnel pour ses enfants. Les transformations apportées par les créateurs de The Ring au scénario original de Ringu et l'attribution du rôle de la mère monstrueuse à une femme au foyer sont emblématiques du traitement que la quasi-totalité des woman's horror films hollywoodiens des années 2000 réservent à ce personnage type.

\section{The Others: femme au foyer, maternité sacrificielle et monstruosité}

La femme au foyer tient le rôle du monstre dans un grand nombre de woman's horror films hollywoodiens des années 2000, dont The Grudge, The Skeleton Key et Drag Me to Hell. Dans The Others, Grace Stewart (Nicole Kidman) est une femme au foyer qui vit seule avec ses deux enfants, Anne (Alakina Mann) et Nicholas (James Bentley), et ses domestiques dans une grande maison victorienne. Son mari est parti au front durant la Seconde Guerre mondiale et n'est plus rentré depuis. Le mode de vie de Grace et de ses enfants est strict: Anne et Nicholas étant allergiques à la lumière, les rideaux sont constamment tirés. Grace est, en outre, dépeinte comme une femme sévère dont la mentalité et la vision du monde sont "archaïques» (elle vit sans électricité, ni radio, ni téléphone). Son refus de s'ouvrir à la modernité est associé à un esprit fermé. Grace est une femme autoritaire qui croit aveuglément au contenu de la Bible et qui s'attend à ce que ses enfants partagent ses convictions. Elle est représentée comme une mère cruelle et dominatrice: contrairement à elle, Nicholas, et surtout Anne, portent un regard critique sur l'univers qui les entoure et sur les enseignements bibliques, ce qui les place dans un rapport de confrontation avec leur mère, qui les punit durement quand ils la contredisent. Le conflit entre mère et fille est de plus nourri par les apparitions successives de fantômes dans la maison: alors qu'Anne établit rapidement un contact avec les créatures surnaturelles, Grace refuse de croire en leur existence bien qu'ils se soient manifestés à elle à plusieurs reprises. Cela conduit à des querelles de plus en plus violentes entre les deux personnages. Le conflit atteint son point culminant quand Grace, croyant

Femme active et femme au foyer dans le woman's horror film américain des années 2000 : ambivalences, contradictions et impasse post-féministe 
qu'Anne est possédée par l'esprit d'une vieille sorcière, tente de l'étrangler.

Malgré qu'on nous la montre comme une femme autoritaire qui peut devenir cruelle et violente vis-à-vis de ses enfants, Grace est également représentée comme une mère surprotectrice qui accourt auprès de son fils et de sa fille dès qu'elle les entend pleurer et qui a constamment peur qu'il leur arrive malheur. Quand, une nuit, les habitants mystérieux de la maison volent les rideaux, laissant Anne et Nicholas exposés à la lumière, Grace se transforme. Devant la perspective de perdre ses enfants, elle accepte immédiatement de croire au surnaturel et entreprend de combattre activement, munie d'un fusil, les créatures qui menacent la vie d'Anne et de Nicholas. Le film se termine cependant sur une révélation inattendue: après la confrontation finale entre les Stewart et "les autres", il s'avère que Grace et ses enfants sont des fantômes. La jeune femme aurait un jour étouffé son fils et sa fille à l'aide d'un oreiller avant de se suicider. Tous les trois auraient ensuite effacé cet événement traumatique de leur mémoire et continué leur vie comme si de rien n'était.

$\mathrm{Au}$ moment où Grace se métamorphose finalement en une bonne mère héroïque, sa monstruosité est révélée au spectateur. Cette révélation fonctionne comme une rédemption pour la jeune femme: en reconnaissant qu'elle est morte et qu'elle a tué ses enfants, Grace accepte de se défaire de son autorité, de sa croyance aveugle dans la Bible et d'avouer à ses enfants qu'elle n'est pas "plus sage» qu'eux". The Others affirme, de ce fait, qu'une femme à l'esprit rigide, qui croit aveuglément en des valeurs traditionnelles "dépassées ", est une mauvaise mère et un monstre et que sa transformation en une bonne mère héroïque dépend de sa capacité à s'ouvrir à ses enfants et à remettre en question sa vision archaïque du monde.

À cause de cette fin inattendue, George Wilson (2006, p. 81-82) considère The Others comme un twist movie - un film à rebondissement final - dont la structure narrative rappelle celle de Jacob's Ladder (Adrian Lyne, 1990), Fight Club (David Fincher, 1999) et surtout The Sixth Sense (M. Night Shyamalan, 1999), où il est question d'un psychiatre, Malcolm 
Crowe (Bruce Willis), qui apprend à la fin du film qu'il est un fantôme. Comme le montre Aviva Briefel (2009, p. 98-99), parce que les twist movies se terminent sur une révélation inattendue, ils invitent le spectateur à les voir une deuxième fois et à les relire à la lumière de la connaissance nouvelle que la découverte finale leur apporte. The Sixth Sense se termine par exemple sur un flashback au cours duquel Malcolm revoit certains moments de sa vie qui prennent tout à coup un sens nouveau pour lui. Cette séquence finale fournit au spectateur des indications sur les passages du film qu'il doit revoir et sur la manière dont il doit les réinterpréter. Dans la séquence finale de The Others, le spectateur apprend que les personnages qu'il croyait vivants sont, en réalité, tous morts, y compris les domestiques engagés par Grace au début du film. Ce passage sert à dévoiler les intentions véritables de madame Mills (Fionnula Flanagan) et de ses deux compagnons, dont les agissements semblaient, jusque-là, étranges et inquiétants. Les trois domestiques s'entêtaient, en effet, à cacher aux Stewart trois tombes qui se trouvaient près de la maison et madame Mills laissait régulièrement entendre qu'il était possible que les enfants aient soudainement guéri. Il s'avère, en fin de compte, que madame Mills et ses compagnons savaient que Grace et ses enfants étaient morts. Leur but était de les amener, en douceur, à prendre conscience de leur condition et de les aider à y faire face. Dans la scène finale du film, le spectateur apprend également la vérité sur l'acte horrifiant commis par Grace, mais le récit ne fournit aucune explication sur les motifs du double infanticide. Cette séquence l'invite implicitement à revoir le film afin, d'une part, de réévaluer sa perspective en fonction de l'attitude de madame Mills et de ses compagnons et en tenant compte de leur relation à Grace et à ses enfants et, d'autre part, de tenter de trouver une explication aux meurtres commis par Grace et à son suicide.

Dans son analyse de The Sixth Sense, Briefel (2009, p. 98106) montre que, quand le spectateur voit le film une deuxième fois en sachant que Malcolm est mort, un décalage cognitif se creuse entre lui et le personnage. Cela le pousse à se distancier de Malcolm. Son savoir nouveau le conduit à examiner minutieusement le comportement du personnage afin de trouver des

Femme active et femme au foyer dans le woman's horror film américain des années 2000 : ambivalences, contradictions et impasse post-féministe 
preuves de sa mort. Ce mécanisme qui, selon elle, est typique des twist movies, ne serait pas à l'œuvre dans The Others parce que le spectateur ne parviendrait jamais véritablement à échapper à la perspective de Grace. Pour appuyer ses propos, elle montre que le film s'ouvre sur la voix off de la jeune femme, qui annonce à ses enfants qu'elle va leur raconter une histoire. Son récit est accompagné par une succession de plans qui montrent des images tirées de livres pour enfants. Ces illustrations anticipent plusieurs scènes du film et suggèrent que Grace connaît déjà la suite de l'histoire qu'elle raconte, qu'elle sait qu'elle et ses enfants sont morts. Même quand le spectateur voit le film pour la deuxième fois, les connaissances qu'il possède sur l'univers fictionnel et l'histoire ne sont pas supérieures à celles de Grace. Il lui est, dès lors, difficile de se distancier du personnage et il continue à s'identifier à la jeune femme. Le spectateur qui voit The Others une deuxième fois cherche davantage une explication au comportement monstrueux de Grace que des preuves que les personnages du film sont en réalité des fantômes. Dans la séquence du générique analysée par Briefel, la question des meurtres commis par la jeune femme ne se pose pas. Dans la séquence qui suit immédiatement, lorsque que Grace se réveille en hurlant d'un cauchemar, la question des meurtres est par contre clairement soulevée. Alors que le spectateur sait qu'elle a probablement rêvé des meurtres qu'elle a commis et que ces événements sont loin d'être le fruit de son imagination, Grace continue pour sa part à croire en sa propre innocence. Nous sommes, dès lors, invités à porter un regard distancié sur la jeune femme et, contrairement à ce qu'affirme Briefel, à nous détacher de sa perspective sur les événements du film.

Le spectateur qui voit The Others pour la deuxième fois se trouve, du reste, dans une position similaire à celle de madame Mills et de ses compagnons. En effet, nous arrivons alors dans l'univers de Grace presque en même temps que les trois domestiques qui frappent à sa porte dans la troisième minute du film et notre intention est la même: nous savons que les Stewart sont morts et nous devons nous mettre à l'écoute de la mère monstrueuse. Notre objectif est de tenter de comprendre pourquoi elle a tué ses enfants, alors que celui de 
madame Mills et de ses compagnons est d'essayer de l'aider à expier son acte et à trouver la paix dans l'autre monde. Le spectateur est invité à se distancier de Grace pour s'identifier à madame Mills, le personnage qui est à la tête du groupe de serviteurs, la seule parmi les trois à bénéficier d'une certaine profondeur psychologique et à partager avec le spectateur le regard qu' elle porte sur la jeune femme. Ce regard est toutefois ambivalent: madame Mills juge Grace et la considère comme une mère cruelle quand elle punit sévèrement et injustement ses enfants, mais elle compatit aussi avec elle dans les moments où Grace lui dévoile ses faiblesses. Le matin de l'arrivée de madame Mills chez elle, la jeune femme lui confie, les larmes aux yeux, qu'elle trouve "insupportable ${ }^{10}$ " son enfermement dans une maison où la lumière ne pénètre jamais. Elle se plaint à plusieurs reprises à la vieille femme de l'isolement auquel la condition de ses enfants l'a réduite: elle ne sort presque jamais de chez elle et n'a aucun contact avec le monde extérieur. Les confessions qu'elle fait à madame Mills permettent de reconstruire son histoire: la famille de Grace a déménagé en 1940, la laissant seule; son mari est ensuite parti faire la guerre, l'abandonnant avec les enfants et, finalement, ses domestiques l'ont quittée. C'est alors qu'elle perd la tête et tue ses enfants avant de se suicider.

Le deuxième visionnement du film, auquel incite le twist final, donne au spectateur une autre image de Grace et lui permet de la percevoir comme une victime dont l'aliénation est attribuable à sa conception sacrificielle de la maternité et à sa fonction de femme au foyer. En effet, l'enfermement et l'isolement de la jeune femme, responsables de sa transformation en mère monstrueuse, sont dus à sa foi en un idéal de maternité qui lui impose de se consacrer entièrement à ses enfants et de renoncer à ses désirs pour leur bien-être, et proviennent de la division traditionnelle des rôles entre les sexes, qui oblige la femme à rester confinée à la maison. The Others condamne doublement le système patriarcal et ses valeurs, qu'il considère comme responsables de la transformation de Grace en une mère criminelle et en une victime aliénée. 


\section{L'« archaïque» femme au foyer}

Le discours de The Ring et The Others sur la femme au foyer s'inspire de celui des féministes de la deuxième vague. Comme l'a montré Imelda Whelehan (2005, p. 63-69), un grand nombre d'ouvrages féministes publiés dans les années 1960 et 1970, dont The Feminine Mystique de Betty Friedan et Diary of a Mad Housewife de Sue Kaufman (1967), mettent l'accent sur le caractère aliénant de la féminité traditionnelle et sur les troubles psychologiques que la fonction de femme au foyer et les tâches qui lui sont associées génèrent chez les femmes. Le woman's horror film des années 2000 critique du reste la situation des femmes dans les sociétés patriarcales traditionnelles en dépeignant le mode de vie de la femme au foyer et le système de valeurs auxquels elle se conforme comme «dépassés » et «archaïques». Dans The Others, elle est doublement enfermée dans un passé révolu: le film, qui déploie une esthétique maniériste et rétro, se passe - du moins le croit-on jusqu'à la révélation finale - dans les années 1940 ; Grace et ses enfants sont morts et sont pour cela à jamais figés dans une époque qui n'est plus. De même, dans The Grudge - le remake américain du film japonais Ju-on (Takashi Shimizu, 2002) —, Kayako (Takako Fuji), le monstre du film, est une femme au foyer japonaise, qui vit dans une société non occidentale, traditionnelle et patriarcale que le film critique vivement: elle était mariée à un homme autoritaire et possessif qu'elle n'aimait pas et qui la tue quand il découvre qu'elle est amoureuse d'un autre homme. Depuis, l'esprit de Kayako hante sa maison et s'en prend à tous ceux qui y pénètrent pour se venger du sort qui lui a été fait. Par opposition à elle, Karen (Sarah Michelle Gellar), la victime/héroïne du film, est dépeinte comme une femme libre: c'est une étudiante américaine qui aspire à intégrer un jour le marché du travail. The Ring, The Others et The Grudge adoptent la vision négative que portent les féministes de la deuxième vague sur le patriarcat et son système de valeurs. Toutefois, en représentant la femme au foyer sous les traits d'un monstre "archaïque", en ancrant la société oppressive dans laquelle elle évolue dans le passé ou dans un ailleurs exotique et en attribuant le rôle de la victime/héroïne qui lutte contre elle à une femme active, américaine et «moderne», le woman's horror 
film disculpe la société américaine contemporaine et la représente comme égalitaire et progressiste.

Pourtant, le woman's horror film ne valorise pas la femme active. Au contraire, ses propos sur la mère qui travaille sont antiféministes. Ce sous-genre tient un discours profondément contradictoire sur la relation de ses personnages féminins à leurs rôles sociaux. Il combine des traits à la fois conservateurs et progressistes: le présent dans ces films est lu à la fois comme un dépassement du passé et de ses valeurs et comme un lieu où sont conservés certains de ses traits. Le woman's horror film fonctionne, à ce titre, comme un texte post-féministe, selon la définition que Valérie Cossy, Fabienne Malbois, Lorena Parini et Silvia Ricci Lempen (Cossy et al. 2009, p. 5-6) avancent de cette notion. Pour elles, sont considérés comme des fictions post-féministes les textes qui portent dans leur discours la «trace de quarante ans de féminisme» et qui, bien qu'ils semblent avoir intégré «la doxa égalitaire du féminisme institutionnel », réinscrivent leurs hérö̈nes dans des schémas traditionnels et brouillent, ce faisant, les frontières entre féminisme et antiféminisme.

\section{Conclusion : femme active versus femme au foyer et impasse post-féministe}

Pour comprendre les enjeux de ces woman's horror films, un détour s'impose par les romans de la chick lit, parangons de la littérature post-féministe. La publication de romans tels que Bridget Jones's Diary d'Helen Fielding (1996) et Sex and the City de Candace Bushnell (1997) et la sortie des films et séries télévisées qui en sont les adaptations ${ }^{11}$ sont généralement associées aux transformations de l'image de la jeune fille dans les médias américains et britanniques des années 1990. En effet, comme l'a montré Anita Harris (2004, p. 6-8), grâce en grande partie au féminisme qui les encourage à prendre leur destin en main en affirmant que "les filles peuvent tout faire ${ }^{12}$ " et que les "filles sont fortes ${ }^{13}$ ", les jeunes femmes font désormais le choix de travailler. L'augmentation considérable du nombre des jeunes filles qui réussissent brillamment leurs études, travaillent et font carrière s'accompagne, dans les sociétés occidentales, de profonds bouleversements sociaux et économiques. Cela a pour 
conséquence de transformer le discours dominant sur la jeune femme active: elle devient un symbole de réussite dans un monde en transformation.

Toutefois, les propos tenus par les fictions populaires américaines et britanniques de la fin des années 1990 et des années 2000 sur la jeune femme active sont ambivalents. Comme l'a montré Imelda Whelehan (2005, p. 162), Bridget Jones's Diary et Sex and the City se centrent sur une ou plusieurs jeunes femmes célibataires qui travaillent et qui font carrière. Elles vivent seules, sont économiquement indépendantes, jouissent d'une certaine aisance matérielle et sont sexuellement libres. Ces jeunes femmes n'ont pas besoin de lutter pour leur émancipation. Au contraire, elles tirent profit des privilèges et des possibilités qu'elles ont acquis grâce à plus de trente ans de lutte féministe.

Toutefois, la majorité d'entre elles ne sont pas heureuses et un gouffre immense semble exister entre les aspirations de leur vie personnelle et celles de leur vie professionnelle, ce qui a pour résultat qu'on représente leur succès comme contribuant à leur misère plutôt que témoignant du fait qu'elles ont dépassé les contraintes sous lesquelles leurs ancêtres féminines ont travaillé (Whelehan 2005, p. 162) ${ }^{14}$.

La multitude d'options dont dispose désormais l'héroïne de ces fictions est perçue comme rendant sa vie plus compliquée. Bien qu'elle évolue dans une société qui semble égalitaire, où elle jouit des mêmes privilèges que les hommes et où le féminisme semble avoir atteint ses buts, au fond d'elle-même, elle ne désire véritablement qu'une seule chose: trouver le prince charmant et fonder une famille. Son histoire se termine, dans la majorité des cas, par un mariage ou la formation d'un couple hétérosexuel.

Malgré que les univers génériques de la chick lit et du film d'horreur soient, a priori, très différents, de même que les destinataires de ces ouvres (des femmes dans le premier cas, un public mixte dans le second), le discours ambivalent tenu par les woman's horror films sur leurs personnages féminins est comparable à celui de la chick lit. Les woman's horror films brouillent les frontières entre féminisme et antiféminisme. Ils considèrent la femme au foyer comme la victime du système patriarcal et regardent le caractère oppressif des rôles féminins traditionnels 
comme responsable de sa transformation en monstre. Ils la représentent, par ailleurs, comme une créature "archaïque» qui n'a plus sa place dans la société américaine contemporaine, dans cette société "égalitaire" où évolue désormais la femme active. Ces films ne font cependant pas l'éloge de la femme qui travaille. Au contraire, ils lui montrent, comme c'est le cas dans The Ring, que son métier et son ambition professionnelle la mèneront à sa perte et feront d'elle une mère négligente qui mettra en danger la santé physique et mentale de ses enfants, de même qu'ils suggèrent que sa métamorphose en héroïne dépend de sa disposition à sacrifier sa carrière pour eux. Ils lui présentent, néanmoins, l'alternative qui s'offre à elle, c'est-à-dire son éventuelle transformation en femme au foyer, comme monstrueuse. Le woman's horror film place, ce faisant, son personnage principal dans une situation impossible: comme c'est le cas dans The Others et Dark Water, un grand nombre de victimes/ héroïnes du genre ne semblent pouvoir trouver leur salut que dans la mort ${ }^{15}$. Parce que le woman's horror film des années 2000 entraîne ses personnages féminins dans une impasse, il rend visible le malaise sous-jacent au post-féminisme qui, tout en partant du principe que le féminisme aurait désormais atteint son but, échoue à présenter des solutions qui remettent en question la féminité traditionnelle.

Université Paris 3

\section{NOTES}

1. «[...] at the center of [their] universe a female who is trying to deal with the emotional, social and psychological problems that are specifically connected to the fact that she is a woman" (traduction de l'auteure, comme toutes les traductions proposées dans le présent article).

2. «[...] it is very rare to find women occupying the narrative and social focus other than as victims."

3. "Second-class citizens of the genre»; «[...] they are there to provide the human ground over which monsters and experts, threat and defender, disordering and ordering impulses can battle it out. "

4. Cf. Friedan 1963.

5. Robin Wood (1986, p. 76) considère Sisters comme «le film d'horreur féministe ultime» («the definitive feminist horror film») et Pauline Kael (2004), l'influente critique de cinéma du magazine The New Yorker, qualifie The Stepford Wives de "premier women's lib gothic» ("The first women's lib gothic») et le compare à Invasion of

Femme active et femme au foyer dans le woman's horror film américain des années 2000 : ambivalences, contradictions et impasse post-féministe 
the Body Snatchers, à la différence qu'il a pour thème principal la libération de la femme.

6. Les recettes de The Ring au box-office s'élèvent à environ 129 millions de dollars et celles de The Others, à environ 96,5 millions de dollars.

7. Comme le montrent les chiffres fournis par le site Internet Infoplease (http://www.infoplease.com/ipa/A0104673.html), aux États-Unis, les femmes font une entrée massive sur le marché du travail à la fin des années 1980, et leur nombre se stabilise ensuite dans les années 1990 et 2000 : alors qu'elles constituaient $38,1 \%$ de la main-d'œuvre américaine en 1970, elles en constituent 42,5\% en 1980, 45,2\% en 1990 et $46,6 \%$ en 2000 .

8. "Comfortable», «sheltered», «protected».

9. «Wiser».

10. "Unbearable».

11. Bridget Jones's Diary (Sharon Maguire, 2001) et Sex and the City (HBO, 19982003).

12. "[...] girls can do anything."

13. "[...] girls are powerful."

14. "However, most of them aren't happy with their lives and there seems to be a huge chasm between the aspirations of their personal lives and those of their professional lives, with the result that their successes are portrayed as contributing to their misery rather than demonstrating that they have moved beyond the constraints under which their foremothers worked."

15. The Grudge, The Skeleton Key et Drag Me to Hell se terminent tous les trois sur la mort du personnage principal féminin.

\section{RÉFÉRENCES BIBLIOGRAPHIQUES}

Basinger 1995: Jeanine Basinger, A Woman's View. How Hollywood Spoke to Women: 1930-1960 [1993], New York, A. Knopf, 1995.

Briefel 2009: Aviva Briefel, "What Some Ghosts Don't Know: Spectral Incognizance and the Horror Film ", Narrative, vol. 17, n 1, 2009, p. 95-110.

Cossy et al. 2009: Valérie Cossy, Fabienne Malbois, Lorena Parini et Silvia Ricci Lempen, "Imaginaires collectifs et reconfiguration du féminisme", dans Valérie Cossy, Fabienne Malbois, Lorena Parini et Silvia Ricci Lempen (dir.), Nouvelles questions féministes. Figures du féminin dans les industries culturelles contemporaines, vol. 28, $\mathrm{n}^{\circ}$ 1, 2009, p. 4-12.

Faludi 1991: Susan Faludi, Backlash. The Undeclared War Against American Women, New York, Crown Publishers, 1991.

Friedan 1963: Betty Friedan, The Feminine Mystique, New York, W.W. Norton \& Company, Inc., 1963.

Goldberg 2004: Ruth Goldberg, "Demons in the Family: Tracking the Japanese "Uncanny Mother Film" from A Page of Madness to Ringu", dans Barry Keith Grant et Christopher Sharrett (dir.), Planks of Reason. Essays on the Horror Film [1990], Revised Edition, Oxford, Scarecrow Press, 2004, p. 370-386.

Harris 2003: Anita Harris, Future Girl. Young Women in the Twenty-First Century, London/New York, Routledge, 2003.

Kael 2004: Pauline Kael, "It's Really a Crock», The Guardian, 19 juillet 2004. http://www.guardian.co.uk/world/2004/jul/19/gender.uk1. D’abord paru dans The New Yorker. 
Martin 2008 : Nina K. Martin, "Dread of Mothering: Plumbing the Depths of Dark Water", Jump Cut: A Review of Contemporary Media, n 50, 2008. http://www. ejumpcut.org/archive/jc50.2008/darkWater/text.html.

Moine 2007 : Raphälle Moine, Remakes. Les films français à Hollywood, Paris, CNRS, 2007.

Tudor 1989: Andrew Tudor, Monsters and Mad Scientists. A Cultural History of the Horror Movie, Oxford, Basil Blackwell, 1989.

Whelehan 2005: Imelda Whelehan, The Feminist Bestseller. From Sex and the Single Girl to Sex and the City, New York, Palgrave Macmillan, 2005.

Wilson 2006: George Wilson, "Transparency and Twist in Narrative Fiction", The Journal of Aesthetics and Art Criticism, vol. 64, n 1, 2006, p. 81-95.

Wood 1986: Robin Wood, Hollywood from Vietnam to Reagan, New York, Columbia University Press, 1986.

\section{ABSTRACT \\ The Active Woman and the Stay-at-Home Woman in the "Woman's Horror Film" of the 2000s in the United States: Ambivalences, Contradictions and Post-Feminist Impasse Pascale Fakhry}

A large number of horror films were released in the 2000s which were also "women's films." These films depict a woman confronted with monstrous creatures, often supernatural in origin, and with specifically female problems, such as motherhood and the work/family clash. Through a detailed analysis of two "woman's horror films" of the period, The Ring (Gore Verbinski, 2003) and The Others (Alejandro Amenábar, 2001), the author first compares the depiction of active women and stay-at-home women and the role of victim, monster or heroine attributed to them by horror narratives. The ambivalence of the woman's horror film's discourse on its leading female characters is then connected to the emergence of post-feminism and its growing influence on popular American culture in the 2000s.

Femme active et femme au foyer dans le woman's horror film américain des années 2000: ambivalences, contradictions et impasse post-féministe 